\title{
The Effect of Remdesivir in Patients with Severe COVID-19, Reporting
}

\section{Case Series}

\author{
Hamid Reza Samimagham ${ }^{1}$, Mehdi Hassani Azad ${ }^{2}$, Mohsen Arabi ${ }^{3}$, Sara Ghazizadeh ${ }^{4}$, Alireza \\ Malektojjari ${ }^{4}$, Meysam Hojjatipour ${ }^{4}$, Dariush Hooshyar (iD) ${ }^{4}$ and Mitra Kazemi Jahromi (iD) ${ }^{5}{ }^{*}$ \\ ${ }^{1}$ Clinical Research Development Center, Shahid Mohammadi Hospital, Hormozgan University of Medical Sciences, Bandar Abbas, Iran \\ ${ }^{2}$ Infectious and Tropical Diseases Research Center, Hormozgan Health Institute, Hormozgan University of Medical Sciences, Bandar Abbas, Iran \\ ${ }^{3}$ Family Medicine Department, Preventive Medicine and Public Health Research Center, Iran University of Medical Sciences, Tehran, Iran \\ ${ }^{4}$ Student Research Comitte, Faculty of Medicie, Hormozgan University of Medical Sciences, Bandar Abbas, Iran \\ ${ }^{5}$ Endocrinology and Metablism Research Center, Hormozgan University of Medical Sciences, Bandar Abbas, Iran \\ "Corresponding author: Endocrinology and Metablism Research Center, Hormozgan University of Medical Sciences, Bandar Abbas, Iran. Email: \\ mitra.kazemijahromi@gmail.com
}

Received 2021 June 14; Revised 2021 June 19; Accepted 2021 November 06.

Keywords: Remdesivir, COVID-19, Severity

\section{Dear editor,}

The outbreak of SARS-CoV-2 in Wuhan, China, in December 2019, which causes COVID-19, has turned into a global health threat to human (1,2). COVID-19 was declared a pandemic by the World Health Organization (WHO) in March 2020 (3). Numerous lives have been affected as a result of compulsory distancing/quarantine. The pandemic can overwhelm national healthcare systems and result in grave consequences for the world's economy if it spreads out of control, or if effective treatments are not used (4). Furthermore, it can incur more harm to people with underlying diseases, especially prevalent diseases such as diabetes and hypertension (5). Clinical trials are being carried out on a wide range of new treatment modalities, but no effective medication has yet been introduced for treating COVID-19 $(6,7)$. Remdesivir (code: GS-5734) is a prodrug monophosphoramidite nucleoside, which was first developed in response to the ebola outbreak in 2014 - 2016 in West Africa $(7,8)$. Remdesivir has shown a wide range of activities against human and zoonotic coronaviruses in preclinical models and proved promising for COVID-19 clinical trials $(7,8)$. This medication has also been assessed in clinical trials with COVID-19 patients $(9,10)$.

The present case series was conducted to assess and compare remdesivir with standard of care (SOC) in terms of effectiveness in patients with severe COVID-19.

This study used records of 60 eligible patients admitted to Shahid Mohammadi Hospital, Bandar Abbas, Iran, in early 2020. All patients underwent a WHO-approved treatment regimen for severe COVID-19. The inclusion cri- teria of the study consisted of presenting to acute respiratory diseases center in Shahid Mohammadi Public Hospital, definitive diagnosis of COVID-19 through positive PCR for SARS-CoV-2, and confirmed clinical and imaging evidence (spiral chest CT-scan). The exclusion criteria were AST or ALT, five times greater than normal, and creatinine clearance $<50 \mathrm{~mL} / \mathrm{min}$ (9). Signs and symptoms required for inclusion (critical categories) (11): Tachypnea (respiratory rate $>30 / \mathrm{min})$, Hypoxemia $\left(\mathrm{PO}_{2}\right.$ saturation $\geq 93, \mathrm{PaO}_{2}$ $<300$ ), lung infiltration ( $<50 \%$ of lung space in 24 to 48 hours), LDH $>245 \mathrm{U} / \mathrm{L}$, progressive lymphopenia, CRP $>$ 100. The study population was divided into two groups of 30 each: Group A receiving remdesivir plus SOC (as per records and treatment process), and group B receiving SOC alone. Remdesivir was administered at a dose of $200 \mathrm{mg}$ on day one and then at $100 \mathrm{mg}$ on days two to ten.

SOC included the following medications according to the treatment protocol of National COVID-19 Committee (9):

(1) Hydroxychloroquine/chloroquine phosphate as hydroxychloroquine sulfate $200 \mathrm{mg}$ tablets or chloroquine phosphate $250 \mathrm{mg}$ tablets (equivalent to $150 \mathrm{mg}$ base value) two tablets bid on the first day, followed by one tablet bd for a minimum of seven days and maximum of 14 days.

(2) One of the following medications at the doctor's discretion:

(A) Two 200/50 mg kaletra (lopinavir/ritonavir) tablets bid after meals for a minimum of seven days and maximum of 14 days. 
(B) One 300/100 mg (atazanavir/ritonavir) tablets daily with food or one $400 \mathrm{mg}$ atazanavir daily for a minimum of seven days and a maximum of 14 days.

The primary objective was to assess the effectiveness of remdesivir + SOC and SOC alone on the patients' recovery and mortality rate. The secondary objective was to assess the difference between the two groups in terms of laboratory findings and hospital stay (Table 1).

Table 1. Difference Between the Two Groups in Terms of Laboratory Findings and Hospital Stay ${ }^{\mathrm{a}}$

\begin{tabular}{|c|c|c|c|}
\hline Variables & $A(N=3)$ & $\mathbf{B}(\mathbf{N}=\mathbf{3})$ & P-Value $^{\text {b }}$ \\
\hline Gender (male) & $17(56)$ & $17(56)$ & 1 \\
\hline Age $(y)$ & $50 \pm 15$ & $51 \pm 13$ & 0.7 \\
\hline $\mathrm{WBC}\left(\times 10^{9} / \mathrm{L}\right)$ & $9000 \pm 2100$ & $9800 \pm 2500$ & 0.34 \\
\hline $\mathrm{Hb}$ g/dL & $11.1 \pm 3.1$ & $11.4 \pm 2.8$ & 0.22 \\
\hline $\mathbf{L D H}(\mathbf{U} / \mathbf{L})$ & $370 \pm 72$ & $384 \pm 90$ & 0.27 \\
\hline CRP $(\mathrm{mg} / \mathrm{dL})$ & $17 \pm 12$ & $19 \pm 14$ & 0.18 \\
\hline Hospital stay (day) & $9 \pm 4$ & $12 \pm 5$ & 0.031 \\
\hline Mortality rate & $6(20)$ & $10(33)$ & 0.002 \\
\hline
\end{tabular}

The present study showed that mortality rate as one of the main outcomes was significantly lower in the group A. Moreover, the mean hospital stay was also significantly less in the group A than that in the group B.

Various studies have reported contradictory results ( 9 , $12,13)$. Thus, further studies are needed to increase scientific, credible, and reliable data. Yet, in agreement with most other studies, the results of the present study confirmed the relationship between administration of interferon and patient recovery. In comparison, considering the effects of different medication groups such as nonsteroidal anti-inflammatory medications like ibuprofen, or medications such as famotidine or $\mathrm{N}$-acetylcysteine, which are effective in managing the inflammatory phase in patients with moderate to severe COVID-19, paying particular attention to the effect of antivirals (with virus-specific action) is highly important in patients with COVID (14-16).

This case series showed that administration of remdesivir in patients with severe COVID-19 has positive effects on reducing the mortality rate and duration of hospital stay. However. Further studies and clinical trials are recommended to confirm these results.

\section{Footnotes}

Authors' Contribution: HR.S and M.KJ contributed to conception, design, and statistical analysis. Other authors contributed to data collection and manuscript drafting. M.KJ supervised the study. All authors approved the final version of the manuscript.

Conflict of Interests: The authors have no conflict of interest.

Ethical Approval: IR.HUMS.REC.1399.499.

Funding/Support: Clinical Research Development Center of Hormozgan University of Medical Sciences, BandarAbbas, Iran, supported this study.

\section{References}

1. Zhu N, Zhang D, Wang W, Li X, Yang B, Song J, et al. A Novel Coronavirus from Patients with Pneumonia in China, 2019. N Engl J Med. 2020;382(8):727-33. doi:10.1056/NEJMoa2001017. [PubMed: 31978945]. [PubMed Central: PMC7092803].

2. Lu R, Zhao X, Li J, Niu P, Yang B, Wu H, et al. Genomic characterisation and epidemiology of 2019 novel coronavirus: implications for virus origins and receptor binding. Lancet. 2020;395(10224):565-74. doi: 10.1016/s0140-6736(20)30251-8.

3. Cucinotta D, Vanelli M. WHO declares COVID-19 a pandemic. Acta Biomedica. 2020:157-60.

4. McKee DL, Sternberg A, Stange U, Laufer S, Naujokat C. Candidate drugs against SARS-CoV-2 and COVID-19. Pharmacol Res. 2020;157:104859. doi: 10.1016/j.phrs.2020.104859. [PubMed: 32360480]. [PubMed Central: PMC7189851].

5. Samimagham HR, Hassani Azad M, Arabi M, Hooshyar D, Sheikhtaheri A, Khorrami F, et al. COVID-19 Severity and Comorbidities in Diabetic Patients. Dis Diagn. 2021;10(3):91-8. doi: 10.34172/ddj.2021.18.

6. Grein J, Ohmagari N, Shin D, Diaz G, Asperges E; Castagna. Compassionate Use of Remdesivir in Covid-19. $N$ Engl J Med. 2020;382(25):2327-36. doi: 10.1056/NEJMc2015312.

7. McCreary EK, Pogue JM. On behalf of the Society of Infectious Diseases Pharmacists, Coronavirus Disease 2019 Treatment: A Review of Early and Emerging Options. Open Forum Infect Dis. 2020;7(4). doi: 10.1093/ofid/ofaa105.

8. Siegel D, Hui HC, Doerffler E, Clarke MO, Chun K, Zhang L, et al. Discovery and synthesis of a phosphoramidate prodrug of a pyrrolo [2, 1 f][triazin-4-amino] adenine C-nucleoside (GS-5734) for the treatment of Ebola and emerging viruses. ACS Publications; 2017.

9. Spinner CD, Gottlieb RL, Criner GJ, Arribas Lopez JR, Cattelan AM, Soriano Viladomiu A, et al. Effect of Remdesivir vs Standard Care on Clinical Status at 11 Days in Patients With Moderate COVID-19: A Randomized Clinical Trial. JAMA. 2020;324(11):1048-57. doi: 10.1001/jama.2020.16349. [PubMed: 32821939]. [PubMed Central: PMC7442954].

10. Beigel JH, Tomashek KM, Dodd LE, Mehta AK, Zingman BS, Kalil AC, et al. Remdesivir for the Treatment of Covid-19 - Final Report. N Engl J Med. 2020;383(19):1813-26. doi: 10.1056/NEJMoa2007764.

11. Gandhi RT, Solomon CG, Lynch JB, del Rio C. Mild or Moderate Covid19. N Engl J Med. 2020;383(18):1757-66. doi: 10.1056/NEJMcp2009249.

12. Wang Y, Zhang D, Du G, Du R, Zhao J, Jin Y, et al. Remdesivir in adults with severe COVID-19: a randomised, double-blind, placebocontrolled, multicentre trial. Lancet. 2020;395(10236):1569-78.

13. Antinori S, Cossu MV, Ridolfo AL, Rech R, Bonazzetti C, Pagani G, et al. Compassionate remdesivir treatment of severe Covid-19 pneumonia in intensive care unit (ICU) and Non-ICU patients: Clinical outcome and differences in post-treatment hospitalisation status. Pharmacol Res. 2020;158:104899. doi: 10.1016/j.phrs.2020.104899. [PubMed: 32407959]. [PubMed Central: PMC7212963]. 
14. Samimagham HR, Hassani Azad M, Haddad M, Arabi M, Hooshyar D, KazemiJahromi M. The Efficacy of Famotidine in improvement of outcomes in Hospitalized COVID-19 Patients: A structured summary of a study protocol for a randomised controlled trial. Trials. 2020;21(1):1-3. doi: 10.1186/s13063-020-04773-6. [PubMed: 33050945]. [PubMed Central: PMC7552598].

15. Rahimi A, Samimagham HR, Azad MH, Hooshyar D, Arabi M, KazemiJahromi M. The efficacy of N-Acetylcysteine in severe COVID-19 pa- tients: A structured summary of a study protocol for a randomised controlled trial. Trials. 2021;22(1):1-3. doi: 10.1186/s13063-021-05242-4. [PubMed: 33845881]. [PubMed Central: PMC8040363].

16. Samimagham HR, Arabi M, Hooshyar D, KazemiJahromi M. The Association of Non-Steroidal Anti-Inflammatory Drugs with COVID-19 Severity and Mortality. Arch Clin Infect Dis. 2020;15(4). doi: 10.5812 /archcid.106847. 